\title{
Perspectivas dos pais sobre dificuldades de APRendizagem ESPECÍFICAS: UM INQUÉRITO POR QUESTIONÁRIO REALIZADO NO NORTE De Portugal
}

\author{
PARENTS' PERCEPTIONS ON SPECIFIC LEARNING DISABILITIES: A QUESTIONAIRE \\ INQUIRY CARRIED OUT IN THE NORTH OF PORTUGAL
}

\author{
Catherine Ruivo BORLIDO ${ }^{1}$ \\ Ana Paula Loução MARTINS²
}

\begin{abstract}
RESUMO: neste artigo apresentaremos as conclusões de um estudo que teve por finalidade conhecer as perspectivas dos pais de alunos do quarto ano do Primeiro Ciclo do Ensino Básico sobre dificuldades de aprendizagem específicas. Os dados foram recolhidos junto de 211 pais numa cidade do norte de Portugal, através de um questionário. As conclusões do estudo mostram que: 1) os pais, na sua maioria, já ouviram ou leram sobre o tema; 2) cerca de um terço dos pais tem pouco conhecimento sobre as condições que estão associadas às dificuldades de aprendizagem específicas; 3 ) a quase totalidade dos pais tem uma atitude de preocupação em relação a um possível diagnóstico de dificuldades de aprendizagem específicas num filho seu; contudo, a cerca de metade dos pais a possibilidade de um filho seu vir ter dificuldades de aprendizagem específicas nunca lhe ocorreu; 4) parece não existir um estigma social negativo perante as dificuldades de aprendizagem específicas; 5) e, a maioria dos pais considera que existe escassez de apoios e recursos destinados às crianças com dificuldades de aprendizagem específicas.
\end{abstract}

PALAVRAS-CHAVE: Educação Especial. Pais. Dificuldades de aprendizagem. Dificuldade específica.

\begin{abstract}
The purpose of this article is to report on the conclusions obtained from a research conducted to investigate parents' perceptions on specific learning disabilities. Participants were 211 fourth grade student's parents. Data were collected in a city in the north of Portugal, using a questionnaire. Results indicate that 1) the vast majority of parents reported having heard or read about the topic of Specific Learning Disabilities; 2) a significant number of parents have no clear understanding of the conditions associated with specific learning disabilities; 3 ) the majority of parents expressed that they would be very concerned if one of their children were found to have specific learning disabilities; but about half of them have never thought that this kind of situation could happen in their family; 4) fewer parents expressed concerns about underlying social stigmas associated with specific learning disabilities; 5) the overwhelming majority of the parents considered that additional resources should be allocated to those children with specific learning disabilities.
\end{abstract}

KEYWORDS: Special Education. Parents. Learning disabilities. Specific Disability.

\section{INTRODUÇÃo}

O campo das dificuldades de aprendizagem específicas ${ }^{3}$ é aquele que, nos últimos anos, tem experimentado mais crescimento de todos os que se inserem no

\footnotetext{
1 Professora de Educação Especial, Portugal. catherine.borlido@gmail.com

2 Professora Auxiliar, Instituto de Educação, Universidade do Minho, Portugal. apmartins@ie.uminho.pt

${ }^{3}$ Segundo Correia (2005, citado por Correia, 2008) as dificuldades de aprendizagem específicas dizem respeito à forma como um indivíduo processa a informação ... e podem, assim, manifestar-se nas áreas da fala, da leitura, da escrita, da matemática e/ou da resolução de problemas, envolvendo défices que implicam problemas de memória, perceptivos, motores, de linguagem, de pensamento e/ou metacognitivos; ... não resultam de privações sensoriais, deficiência mental, problemas motores, défice de atenção, perturbações emocionais ou sociais, embora
} 
contextodasnecessidadeseducativasespeciais(CORREIA, 2008). Internacionalmente, muitos profissionais do campo da medicina, da psicologia e da educação contribuíram para esse crescimento. Contudo, são os pais quem, segundo Hallahan e Mercer (2002), tiveram um papel fulcral na emergência deste campo nos EUA. De fato, em 6 de Abril de 1963, um conjunto de pais norte-americanos, preocupados com a escassez de programas educativos que fizessem face aos problemas dos seus filhos, reuniu-se numa conferência que denominaram Exploration Into the Problems of the Perceptually Handicapped Child (KIRK, 1970; WIEDERHOLT, 1974; CRUICKSHANK, 1981; CORREIA, 1991; 1992; KAVALE; FORNESS, 1995; HALLAHAN; MERCER, 2002). Um dos principais objetivos desta conferência era encontrar um termo que se aplicasse, não só a um determinado conjunto de crianças, mas que servisse, também, de denominação para a associação nacional que os pais pretendiam fundar (KIRK, 1963). Foram convidados vários oradores, entre eles Myklebust, Kephart e Kirk (KIRK, 1970). Segundo Hallahan e Mercer (2002), Kirk destacou-se de entre todos, ao fazer um discurso que ficou para a história por introduzir, no domínio público, o termo e a definição de dificuldades de aprendizagem específicas. De fato, esta conferência, em especial o discurso de Kirk, teve efeitos surpreendentes. O campo das dificuldades de aprendizagem específicas tinha, então, um nome genérico que podia abarcar uma variedade de problemas acadêmicos, e uma organização de pais e de profissionais dedicada à defesa dos interesses dos indivíduos com esta problemática (HAMMILL, 1993). Pode dizer-se, com alguma certeza, que o campo das dificuldades de aprendizagem específicas, enquanto entidade, começou nessa altura nos EUA (KIRK, 1981; HAMMILL, 1993; KAVALE; FORNESS, 1995). Após esta conferência, várias associações de profissionais e de pais desenvolveramse e desempenharam um papel primordial, tanto na elaboração de definições como na advocacia. Em Portugal, no que respeita os alunos com dificuldades de aprendizagem específicas, até aos anos 90, não surgiu nenhuma associação deste tipo e os pais se deparavam com a falta de respostas efetivas por parte do Estado. Esta realidade levou, no ano 2000, à criação da Associação Portuguesa de Dislexia e, em 2007, à Associação Portuguesa de Pessoas com Dificuldades de Aprendizagem Específicas. Desta forma, ambas, constituídas por pais e profissionais interessados na problemática, tiveram a sua origem na falta de serviços de apoio. Têm tido como missão informar e sensibilizar os indivíduos com dificuldades de aprendizagem específicas, os pais e a sociedade civil em geral, advogar pelos direitos das crianças, jovens e adultos, encorajar a investigação, disseminar informação e promover a formação de profissionais.

Tendo por base o contexto, tanto internacional como nacional, referente ao papel dos pais, parece-nos importante que conheçamos a sua maneira de considerar, de ver, de encarar o tema. Como tal, desenvolvemos este estudo, conscientes de quatro aspectos. Primeiro, dos resultados de um estudo realizado pelo Worldwide Roper Starch para a Emily Tremaine Foundation, em Março de 2000,

exista a possibilidade de estes ocorrerem em concomitância com elas, podem, ainda, alterar o modo como o indivíduo interage com o meio envolvente. (p.46-47) 
nos EUA, que revelou que dois terços dos norte-americanos adultos relacionavam as dificuldades de aprendizagem específicas com a deficiência intelectual; que existia, ainda, uma percentagem elevada de pessoas que acreditavam que as dificuldades de aprendizagem específicas estão relacionadas com o ambiente em que a criança está inserida, ou com problemas emocionais; e outras, ainda, que consideravam que este tipo de dificuldades acadêmicas era fruto de preguiça. Conscientes, em segundo lugar, do pouco conhecimento que a sociedade civil nacional pode ter sobre o tema. Conscientes, ainda, de que a incompreensão do conceito de dificuldades de aprendizagem específicas, por parte dos pais e dos profissionais, é um dos fatores responsáveis pelo insucesso escolar de muitos alunos que apresentam este tipo de necessidades educativas especiais (CORREIA; MARTINS, 1999). Finalmente, conscientes de que os pais dos alunos com dificuldades de aprendizagem específicas devem contar com o apoio e os conhecimentos dos outros pais no processo de advocacia. A finalidade deste estudo foi, assim, descrever e analisar as perspectivas que os pais dos alunos do quarto ano do Primeiro Ciclo do Ensino Básico de uma cidade do norte de Portugal têm sobre dificuldades de aprendizagem específicas.

\section{MÉTodo}

Este estudo de natureza quantitativa, do tipo descritivo e inferencial apresenta um desenho metodológico que caracterizamos de seguida.

\subsection{Amostra}

A amostra é constituída por 211 dos 217 pais de alunos do quarto ano do Primeiro Ciclo do Ensino Básico das quatro escolas existentes numa cidade do norte de Portugal; ou seja, a amostra representa 97,2\% da população. Dos 211 participantes, 34,8\% são do sexo masculino e $65,2 \%$ do feminino. A idade dos pais está distribuída entre os 18 e os 24 anos (1,4\%), os 25 e os 34 anos (14,4\%), os 35 e os 44 anos (73,6\%), os 45 e os 54 anos (8,2\%) e aqueles com mais de 55 anos $(2,4 \%)$.

No que refere às habilitações acadêmicas, os participantes têm, maioritariamente, o $12^{\circ}$ ano $(33,8 \%)$ e a licenciatura ou bacharelato $(28,5 \%)$. No que diz respeito às profissões dos pais, 34,3\% têm profissões do tipo vendedor, polícia, ajudante da ação direta, monitor ou empregado de balcão, 13,5\% são administrativos, 9,7\% professores, 8,7\% estão desempregados, 1,9\% são bancários e $1 \%$ fisioterapeutas. Relativamente ao rendimento, quase três quartos dos pais $(74,5 \%)$ responderam que recebem um vencimento mensal fixo.

\subsection{INSTRUMENTO DE RECOLHA E ANÁLISE DE DADOS}

Neste estudo, foi utilizado como instrumento de recolha de dados o questionário de perguntas fechadas desenvolvido pela Emily Hall Tremaine 
Foundation (2000), nos EUA, e traduzido e adaptado para o contexto português por Araújo (2009), com a autorização da referida Fundação.

O questionário é constituído por três partes distintas. A primeira parte é constituída por uma introdução que explica aos participantes o objetivo do estudo, garante a confidencialidade e anonimato das respostas, indica o tempo estimado de resposta e, finalmente, agradece a sua disponibilidade para participar. A segunda parte apresenta um conjunto de 20 questões, num total de 78 itens de resposta fechada, em que as respostas são codificadas segundo uma escala de Likert. O conjunto das questões colocadas aos pais encontra-se dividido em cinco categorias: familiarização, condições associadas, atitudes e comportamentos, estigma e, finalmente, apoios e recursos. Por fim, a terceira parte do questionário é constituída por um conjunto de questões relacionadas com os dados demográficos dos participantes.

Orientamos o estudo com o objetivo de descrever as perspectivas dos pais no que respeita as cinco categorias anteriormente mencionadas. Quisemos, ainda, conhecer o impacto das variáveis gênero, idade, habilitações acadêmicas, profissões e classes de rendimento dos participantes.

\subsection{Procedimentos de Recolha de Dados}

A recolha de dados foi efetuada após as devidas autorizações, com a colaboração quer dos professores, quer dos diretores de turma, a quem foi explicada qual a relevância do estudo e a razão porque era vital os pais participarem. Assim, estes profissionais disponibilizaram-se a entregar aos pais dos alunos os questionários, acompanhados de uma carta explicativa e de agradecimento. Ao longo de dois meses, os questionários preenchidos foram sendo entregues em cada uma das escolas participantes.

\subsection{Procedimentos de análise de dados}

Neste estudo, definimos hipóteses estatísticas referentes à existência de diferenças significativas no que respeita as variáveis independentes gênero, idade, habilitação acadêmica, profissão e rendimento dos pais, nos resultados obtidos em cada uma das cinco categorias do questionário.

Para testar as hipóteses anteriormente mencionadas, recorremos à estatística inferencial, nomeadamente aos testes paramétricos $t$-Student para amostras não relacionadas, à Análise de Variância (ANOVA) e a procedimentos de comparação múltipla (Tukey pós-hoc). O nível de significância utilizado para rejeitar a hipótese nula foi $p<0,05$. Paralelamente, para a análise descritiva usaram-se frequências e médias. 


\section{Resultados E discussão}

De seguida, apresentamos uma síntese descritiva e inferencial dos resultados obtidos nesteestudo em cada uma das cinco categorias que constituem o instrumento de recolha de dados. Sempre que se justifique, fazemos uma discussão destes resultados, tendo por base o estudo elaborado pela Roper Starch Worldwide Inc, em 2000, para a Emily Hall Tremaine Foundation, bem como a literatura nacional e internacional.

\subsection{A MAIOR PARTE DOS PAIS CONSIDERA ESTAR FAMILIARIZADA COM O TEMA DAS DIFICULDADES DE APRENDIZAGEM ESPECÍFICAS:}

- $91,9 \%$ dos pais diz já ter ouvido falar sobre o tema das dificuldades de aprendizagem específicas. Os resultados de ANOVA indicam que existem diferenças estatisticamente significativas na variável profissão no que respeita à familiaridade com o tema, $\operatorname{com} F=3,149, p=0,001$. O teste post hoc Tukey mostra que essas diferenças se encontram entre os pais professores $(M=1,65)$ e os que têm "outras profissões" $(M=1,74)$, para $\mathrm{p}=0,000$, sendo que são os professores os que mais ouviram falar do assunto;

- 56,4\% dos pais diz já ter lido sobre dificuldades de aprendizagem específicas. Os resultados de análise de variância apontam para diferenças estatisticamente significativas na variável profissão no que respeita esta questão, $\operatorname{com} F=2,305$, $p=0,01$. O teste post hoc Tukey mostra que essas diferenças existem entre os pais que são professores $(M=2,55)$ em relação aos pais que são administrativos $(M=3,39)$, que têm profissões de trabalho manual $(M=3,55)$, que estão desempregados $(M=3,50)$ ou que exercem "outras profissões" $(M=3,32)$, para $p<0.05$. São os pais professores e os pais que exercem profissões manuais que, respectivamente, admitem ter lido mais e menos sobre o tema.

Estes resultados são semelhantes aos obtidos no estudo desenvolvido pelo Roper Starch Worldwide Inc (2000), que reportou que $84 \%$ dos pais norte-americanos tinham ouvido falar do tema e, tal como no nosso estudo, não sobressaíram diferenças de gênero. É de sublinhar que, em relação aos pais portugueses, 54,3\% nunca conheceu alguém com este tipo de necessidades educativas especiais.

3. 2 OS PAIS TÊM POUCO CONHECIMENTO SOBRE AS CONDIÇÕES QUE ESTÃo ASSOCIADAS ÀS DIFICULDADES DE APRENDIZAGEM ESPECÍFICAS:

- $51 \%$ dos pais associa as dificuldades de aprendizagem específicas à deficiência intelectual, $29,4 \%$ à deficiência visual e 32,2\% à deficiência auditiva. Apenas $37,4 \%$ acredita que estas crianças ou jovens têm uma inteligência na média ou acima da média. Estes resultados portugueses corroboram os do Roper Starch Worldwide Inc (2000), que obtiveram uma associação à deficiência intelectual de $56 \%$, à deficiência visual de $31 \%$ e à deficiência auditiva de $36 \%$. Segundo as definições conceptuais mais utilizadas (HAMMILL, 1990), as dificuldades 
de aprendizagem específicas podem ocorrer concomitantemente com outras condições, mas não são provocadas por elas; ou seja, esta problemática não é uma causa primária de necessidades educativas especiais de âmbito sensorial (visual ou auditivo), intelectual, motor, de distúrbios emocionais, de desvantagem cultural, econômica ou social ou de um ensino insuficiente ou inadequado (HALLAHAN et al., 2005; CORREIA, 2007; CORREIA, 2008);

- Para 54\% dos pais, a dislexia está associada às dificuldades de aprendizagem específicas, indicando os resultados do teste ANOVA que existem diferenças estatisticamente significativas nas respostas ao nível das habilitações acadêmicas, com $F=3,655, p=0,002$. O teste post hoc Tukey mostra que existem diferenças estatisticamente significativas entre os pais que têm o $9^{\circ}$ ano $(M=2,35)$ e os que têm a licenciatura/bacharelato $(M=1,59)$, com $p=0,029$, sendo que os últimos são aqueles que mais fazem esta associação. De fato, a dislexia é uma dificuldade de aprendizagem específica (HALLAHAN et al., 2005; SHAYWITZ; SHAYWITZ, 2005) e está presente em cerca de $80 \%$ dos alunos que constituem este grupo (HALLAHAN et al., 2005);

- Seis em cada dez pais $(63,5 \%)$ discordam total ou parcialmente com a afirmação que indica que dificuldades de aprendizagem específicas são uma condição vitalícia. A investigação tem mostrado que, para a maioria dos indivíduos, esta é, de fato, uma condição para toda a vida, embora com manifestações que variam em função do seu estado de desenvolvimento (na medida em que a pessoa vai aprendendo a lidar com os seus problemas) e das exigências do meio escolar e profissional (KAVALE, 1988; HALLAHAN et al., 1999; GERBER, 2001; HALLAHAN et al., 2005).

3.3 A quase totalidade dos pais tem uma atitude de preocupação em relação a UM POSSÍVEL DIAGNÓSTICO DE DIFICULDADES DE APRENDIZAGEM ESPECÍFICAS NUM FILHO SEU; CONTUDO, A CERCA DE METADE DOS PAIS A POSSIBILIDADE DE UM FILHO SEU VIR TER DIFICULDADES DE APRENDIZAGEM ESPECÍFICAS NUNCA LHE OCORREU:

- A grande maioria dos pais $(92,4 \%)$ reagiria com muita ou alguma preocupação se fossem diagnosticadas dificuldades de aprendizagem específicas ao seu filho. Os resultados do teste t-Student indicam diferenças estatisticamente significativas entre as respostas do gênero masculino $(M=1,83)$ e as do gênero feminino $(M=1,41)$, $\operatorname{com} t(207,102)=2,723$ para $p=0,008$, sendo que são as mães as que indicaram reagir com mais preocupação.

- Cerca de metade dos pais indicou que nunca lhe ocorreu que um filho seu pudesse ter um problema sério com a aprendizagem na escola $(59,3 \%)$ ou com os trabalhos de casa $(58,4 \%)$. Existem mais homens a responder que nunca lhes ocorreu nenhuma destas situações e, consequentemente, mais mães a responder "sim". São os bancários quem mais responde que nunca lhe ocorreu qualquer uma das situações. Quanto às idades, são os pais com mais de 55 anos quem 
mais dá o "não" como resposta. Estes resultados são muito semelhantes aos do estudo realizado nos EUA (2000), já que quatro em 10 pais norte-americanos $(40 \%)$ indicaram ter já pensado que os filhos podiam ter um problema sério com a aprendizagem na escola ou os trabalhos de casa (ROPER STARCH WORLDWIDE INC., 2000). Segundo Martins (2006), muitos pais não suspeitam que o seu filho ou filha apresenta dificuldades de aprendizagem específicas, se não dois, três ou quatro anos depois do início da escolaridade, uma vez que estes têm um desenvolvimento considerado dentro dos padrões normais em áreas não acadêmicas, ao contrário de outras necessidades educativas especiais, que são geralmente diagnosticadas logo após o nascimento ou nos primeiros anos de vida.

\subsection{Não PARECE EXISTIR UM ESTIGMA SOCIAL NEGATIVO ASSOCIAdo Às DifiCULDAdES DE APRENDIZAGEM ESPECÍFICAS:}

- Se suspeitassem que o seu filho tinha dificuldades de aprendizagem específicas, $45 \%$ dos pais pedia ajuda aos professores dos filhos e 49,8\% pedia essa ajuda fora do sistema escolar mas com o conhecimento dos professores do filho. Uma minoria tomaria outras atitudes, como procurar ajuda fora do sistema escolar sem o conhecimento do professor $(2,4 \%)$, não faria nada $(1,4 \%)$, ou não sabia o que fazer (1,6\%). No estudo de Roper Starch Worldwide Inc (2000), em relação à mesma questão, 72\% dos pais falaria com os professores do filho/filha, 25\% procuraria apoio fora da escola sem envolver os professores do filho/filha.

- Os pais referem que, se soubessem que um filho/filha seu tinha dificuldades de aprendizagem específicas, informariam e analisavam o assunto com o professor $(94,3 \%)$, procurariam ajuda de um psicólogo $(83,9 \%)$, discutiriam o assunto em família $(79,1 \%)$ e procurariam ajuda médica $(74,4 \%)$. As hipóteses de procurar na internet um grupo de discussão sobre o assunto (46,9\%) e de discutir o assunto com amigos (42,2\%) também são contempladas. De realçar, ainda, que 10\% dos pais não diria nada a ninguém. Os resultados do teste $t$-Student para amostras independentes denotaram diferenças estatisticamente significativas entre a cotação dos pais $(M=1,68)$ e a das mães $(M=1,12)$ relativamenteà atitude de discutir com amigos se soubessem que um filho tivesse dificuldades de aprendizagem específicas, com $t(208,158)=-2,005$ para $p=0,046$, sendo as mães quem mais o faria. Os resultados do teste ANOVA indicam diferenças estatisticamente significativas entre as respostas dos pais segundo a sua profissão, relativamente à atitude de discutir com amigos se soubessem que um filho tivesse dificuldades de aprendizagem específicas, $\operatorname{com} F=2,775, p=0,002$. O teste post hoc Tukey indica que as diferenças se encontram (a) entre a profissão de dona de casa $(M=2,50)$ e a de professor $(M=1,40)$, com $p=0,004$, sendo que são os professores quem mais admite discutir o assunto com amigos; e (b) entre a profissão de dona de casa $(M=2,50)$ e a de profissional liberal $(M=1,53)$, com $p=0,047$, sendo os últimos quem mais admite discutir o assunto com amigos. 
No estudo de Roper Starch Worldwide Inc (2000), em relação à mesma questão, $67 \%$ dos pais falaria com o médico ou pediatra de família; $66 \%$ consultaria os professores, 45\% procuraria apoio de psicólogos/, psiquiatras, 54\% a biblioteca, $39 \%$ a livraria e $34 \%$ a internet.

\subsection{A MAIORIA DOS PAIS CONSIDERA QUE EXISTE ESCASSEZ DE APOIOS E RECURSOS DESTINADOS ÀS CRIANÇAS COM DIFICULDADES DE APRENDIZAGEM ESPECÍFICAS:}

- Somente 9\% dos pais pensam existirem recursos suficientes para apoiarem as crianças com dificuldades de aprendizagem específicas, sendo que são os homens quem mais concorda com a afirmação;

- Três em cada quatro pais $(74,8 \%)$ consideram que o governo não atribui financiamento suficiente para a educação dos alunos com dificuldades de aprendizagem específicas. Os pais que mais dão esta resposta, segundo a profissão, são os empresários. Mais de metade dos pais $(52,9 \%)$ dedicaria mais recursos para o apoio a estes alunos.

- A maioria dos pais éa favor dequeas crianças com dificuldades de aprendizagem específicas usufruam de adaptação ao nível do tempo $(73,8 \%)$ e do ambiente $(69,5 \%)$ para a realização de testes. Quase metade $(49,5 \%)$ concorda que todas as crianças deveriam fazer os mesmos testes, independentemente de terem ou não dificuldades de aprendizagem específicas. São os homens com o $9^{\circ}$ ano e com idades compreendidas entre os 18 e os 25 anos quem mais concorda com esta situação. Os resultados do teste $t$-Student indicam diferenças estatisticamente significativas nas respostas dos pais $(M=2,59)$ e das mães $(M=2,01)$ para a opinião sobre se as crianças com dificuldades de aprendizagem específicas deveriam ter mais tempo para realizarem os testes, com $t(208,116)=3,665$ para $p=0,000$, sendo que são as mães quem mais concorda com essa ideia. No estudo de Roper Starch Worldwide Inc (2000), 76\% dos pais concorda com tempo extra para a realização de testes.

\section{Conclusões}

Existe uma grande quantidade de autores de vários países que defende que a participação dos pais é fulcral na educação (FLANAGAN, 2001). Também em Portugal, essa ideia encontra-se já bem enraizada, e como referem, por exemplo, Vilhena e Silva (2002), os pais e a comunidade em geral têm como papel estarem abertos à discussão e sugerir recursos materiais e humanos para que todos fiquem a lucrar com a sua participação. Fica, portanto, claro que, para além do papel dos progenitores ser importante na envolvência e participação da contingência dos seus filhos, é um papel também essencial, não só a nível familiar como também social (STRECHT, 2008). A realização deste estudo mostrou que a maior parte dos pais considera estar familiarizada com o tema das dificuldades de aprendizagem 
específicas; que os pais têm pouco conhecimento sobre as condições que estão associadas às dificuldades de aprendizagem específicas; a quase totalidade dos pais tem uma atitude de preocupação em relação a um possível diagnóstico de dificuldades de aprendizagem específicas num filho seu; a cerca de metade dos pais a possibilidade de um filho seu vir ter dificuldades de aprendizagem específicas nunca lhe ocorreu; que parece não existir um estigma negativo perante as dificuldades de aprendizagem específicas; e que a maioria dos pais considera que existe escassez de apoios e recursos destinados às crianças com dificuldades de aprendizagem específicas. Considerando que a investigação tem evidenciado que a problemática das dificuldades de aprendizagem é universal, ou seja, ocorre em todas as línguas, culturas e nações do mundo (LERNER, 2000), sublinhamos a semelhança entre os resultados dos portugueses e dos norte-americanos.

Estes resultados podem impulsionar a discussão de ideias entre pais e profissionais e contribuir, englobados num conjunto alargado de investigação, para "o debate, para a produção de conhecimento e para a melhoria da vida dos alunos com dificuldades de aprendizagem específicas, das suas famílias e dos profissionais envolvidos, bem como para o desenvolvimento de uma sociedade mais informada e formada" (MARTINS, 2006).

\section{REFERÊNCIAS}

ARAÚJO, A. M. L. Perspectivas dos pais de alunos do $4 .^{\circ}$ ano do ensino básico sobre dificuldades de aprendizagem específicas: Um inquérito por questionário realizado na cidade de Braga. 2009. Mestrado (Educação Especial, especialização em dificuldades de aprendizagem específcas) - Instituto de Educação Universidade do Minho, Braga, 2009.

CORREIA, L. M. O que são as dificuldades de aprendizagem? Revista Portuguesa de Educação, v.4, n.1, p.91-109, 1991.

. Dificuldades de aprendizagem: uma perspectiva histórica. Lisboa: Escola Superior de Educação de Lisboa, 1992.

. Para uma definição portuguesa de dificuldades de aprendizagem específicas. Revista Brasileira de Educação Especial, Marília, v.13, n.2, p.155-172, 2007.

. Dificuldades de aprendizagem específicas: Contributos para uma definição portuguesa. Porto: Porto Editora, 2008.

CORREIA, L. M.; MARTINS, A. P. L. Dificuldades de aprendizagem: O que são? Como entendê-las? Porto: Porto Editora, 1999.

CRUICKSHANK, W. M. Concepts in learning disabilities: Selected writings. Syracuse, NY: Syracuse University Press, 1981.

GERBER, P. J. Learning disabilities: A life-span approach. In: HALLAHAN, D. P.; KEOGH, B. (Ed.). Research and global perspectives in learning disabilities: Essays in honor of William M. Cruickshank. Mahwah, NJ: Lawrence, 2001. p.167-180.

HALLAHAN, D. P.; KAUFFMAN, J. M.; LLOYD, J. W. Introduction to learning disabilities. Boston: Allyn and Bacon, 1999. 
HALLAHAN, D. P. et al. Introduction to learning disabilities: Foundations, characteristics, and effective teaching. Boston: Allyn and Bacon, 2005.

HALLAHAN, D. P.; MERCER, C. D. Learning disabilities: Historical perspectives. In: BRADLEY, R.;DANIELSON, L., et al (Ed.). Identification of learning disabilities: Research to practice. Mahwah, NJ: Erlbaum, 2002. p.1-65.

HAMMILL, D. D. On defining learning disabilities: An emerging consensus. Journal of Learning Disabilities, v.23, p.74-84, 1990.

. A brief look at the learning disabilities movement in the United States. Journal of Learning Disabilities, v.26, p.295-310, 1993.

KAVALE, K. A. Status of the field: Trends and issues in learning disabilities. In:

KAVALE, K. A. (Ed.). Learning disabilities: State of the art and practice. Boston: CollegeHill Press, 1988. p.3-21.

KAVALE, K. A.; FORNESS, S. R. The nature of learning disabilities: Critical elements of diagnosis and classification. Mahwah, NJ: Erlbaum, 1995.

KIRK, S. A. Behavioral diagnosis and remediation of learning disabilities. In:

ANANYMOUS, PROCEEDINGS OF THE ANNUAL MEETING OF THE CONFERENCE ON EXPLORING INTO THE PROBLEMS OF THE PERCEPTUAL HANDICAPPED CHILD, 1963. Chicago. Perceptually Handicapped Children. p.1-7.

. Reflections and comments on learning disabilities. Learning disabilities in the decade of the 70s: A statement of position for the whit house conference on children and youth, Public Health Service Publication No. 1415. Department of Health, Education, and Welfare. Washington, D.C. 1970.

. Learning disabilities: A historical note. Academic Therapy, v.17, p.5-11, 1981.

LERNER, J. W. Learning disabilities: Theories, diagnosis, and teaching strategies. 8th. Boston: Houghton Mifflin, 2000.

MARTINS, A. P. L. Dificuldades de aprendizagem: Compreender o fenómeno a partir de sete estudos de caso. 2006. Doutorado (Educação Especial) - Instituto de Estudos da Criança, Universidade do Minho, Braga, 2006.

ROPER STARCH WORLDWIDE INC. Measuring progress in public E parental uniderstanding of learning disabilities. Emily Hall Tremaine Foundation, Inc., Hartford, CT. 2000.

SHAYWITZ, S. E.; SHAYWITZ, B. A. Dyslexia (Specific Reading Disability). Biological Psychiatry, v.57, n.11, p.1301-1309, 2005. Disponível em: <http:/ /www.sciencedirect. com/science/article/B6T4S-4FWT41F-1/2/a6fb9149fbb6b1e66d36a8b40f943841 >.

WIEDERHOLT, J. L. Historical perspectives on the education of the learning disabled. In: MANN, L.; SABATINO, D. A. (Ed.). The second review of special education. PhiWiederholt, J. L.ladelphia: JSE Press, 1974. p.103-152.

Recebido em: 23/10/2011

Aprovado em: 06/12/2011 